\title{
Quantum Teleportation with an Accelerated Partner in Open System
}

\author{
Maohuai Xiang, Jiliang Jing \\ Department of Physics, and Key Laboratory of Low Dimensional Quantum Structures \\ and Quantum Control of Ministry of Education, Hunan Normal University, Changsha, China \\ Email: "jljing@hunnu.edu.cn
}

Received September 23, 2012; revised November 5, 2012; accepted November 14, 2012

\begin{abstract}
We investigate the teleportation between two relatively accelerating partners undergoing the phase flip, bit flip and bit-phase flip channels. We find that: 1) the fidelity decreases by increasing the acceleration of accelerated observer; 2) the dynamic evolution of the fidelity is different for various channels if the acceleration is fixed; and 3) the fidelity is always symmetric about $\beta^{2}=\frac{1}{2}$ where $\beta$ is a parameter of the transmission state.
\end{abstract}

Keywords: Quantum Teleportation; Non-Inertial Frames; Open System

\section{Introduction}

In recent years, quantum mechanics and quantum infornmation science have developed rapidly. As one of the significant characteristics of quantum mechanics [1], the quantum entanglement has been attracted much attention [2] and its momentous application is quantum teleportation proposed by Bennett et al. [3]. This application involves many attractive theoretical features and shows a bran-new way unlike classical teleportation [4-8]. However, the early study was confined to the inertial frame. About ten years ago, P. M. Alsing et al. considered the teleportation with an uniformly accelerated partner $[9,10]$, which firstly extended the quantum information to the non-inertial frames. Since then, many authors focus on this interdiscipline [11-16]. Moreover, some of them studied the teleportation in some kinds of black hole spacetimes and discussed how the Hawking effect affects the entanglement and teleportation [17-21]. It is not doubtful that these studies will makes the theories more complete, not only for quantum information theory, but also for relativistic quantum mechanics and quantum field in curved spacetime.

It is well known that the interaction between quantum system and surrounding environment is inevitably in our real world, and then the dynamic evolution of the quantum system is non-unitary (although the whole system including the quantum system and surrounding environment evolves in an unitary fashion), so it becomes more complex. Generally, this interaction can be viewed as the

${ }^{*}$ Corresponding author. interchange of information between quantum system and surrounding environment. It plays a fundamental role in the description of the quantum-to-classical transition $[22,23]$ and has been successfully applied in some important places such as the cavity QED [24] and ion trap experiments [25]. So studying teleportation between two relatively accelerated partners in open system is an interesting topic, which not only makes the theory of the quantum teleportation more complete, but also is helpful for us to understand how the surrounding environment affects the quantum teleportation. In this paper, we will discuss teleportation for fermionic resources with one of the EPR partners accelerated undergoing the environment, such as the bit flip, the phase flip, and the phase-bit flip channels.

The outline of this paper is as follows. In Section 2 we analyse the entangled state shared by two relatively accelerated partners. In Section 3 we introduce the dynamics of the system interacting with the environment. In Section 4 we investigate how the environment effects the fidelity of the teleportation when teleportation undergoes environment. And we summarize and discuss conclusions in the last section.

\section{Entangled State between Alice and Rob}

We assume that an unknown state is teleported from Alice who stays rest to Rob who moves with uniform acceleration, and all our work is confined to Dirac field. As shown in Refs. [26-29], the corresponding Unruh spinor basis states can be described by a superposition of Unruh 
monochromatic modes

$$
|0\rangle_{U}=\underset{\omega}{\otimes}\left|0_{\omega}\right\rangle_{U},|1\rangle_{U}=\underset{\omega}{\otimes}\left|1_{\omega}\right\rangle_{U},
$$

with

$$
\begin{aligned}
& \left|0_{\omega}\right\rangle_{U}=\cos r\left|0_{\omega}\right\rangle_{I}\left|0_{\omega}\right\rangle_{I I}+\sin r\left|1_{\omega}\right\rangle_{I}\left|1_{\omega}\right\rangle_{I I}, \\
& \left|1_{\omega}\right\rangle_{U}=\left|1_{\omega}\right\rangle_{I}\left|0_{\omega}\right\rangle_{I I},
\end{aligned}
$$

where the subscripts $I$ and $I I$ represent Rindler regions $I$ and $I I, \cos r=\left(\mathrm{e}^{-2 \pi \omega c / a}+1\right)^{-1 / 2}, \quad a$ is Rob's acceleration, $\omega$ is the frequency of the Dirac particle, and $c$ is the speed of light in vacuum.

Considering Alice and Rob initially share the maximally entangled Bell state

$$
|\Phi\rangle=\frac{1}{\sqrt{2}}\left(|0\rangle_{A}|0\rangle_{R}+|1\rangle_{A}|1\rangle_{R}\right),
$$

then Alice stays stationary, while Rob moves with an uniform acceleration. To describe the state shared by them, we must rewrite the initial state (3). Using Equation (2), the state can also be represented as

$$
\begin{aligned}
\rho_{A R}= & \frac{1}{2}\left(\cos ^{2} r|000\rangle\langle 000|+\cos r \sin r| 000\rangle\langle 011|\right. \\
& +\cos r|000\rangle\langle 110|\cos r \sin r| 011\rangle\left\langle 000\left|+\sin ^{2} r\right| 011\right\rangle\langle 011| \\
& +\sin r|011\rangle\langle 110|\cos r| 110\rangle\langle 000|+\sin r| 110\rangle\langle 011|+\sin r| 110\rangle\langle 110|) .
\end{aligned}
$$

As we all known, the regions $I$ and $I I$ of Rindler spacetime are causally disconnected, and our accelerated observer must remain in either region $I$ or $I I$. Thus, for Rob who stays in region $I$ we must trace over the modes in region II where he can't access. After taking the trace, Equation (4) in terms of matrix turns to be

$$
\rho_{A R_{I}}=\frac{1}{2}\left(\begin{array}{cccc}
\cos ^{2} r & 0 & 0 & \cos r \\
0 & \sin ^{2} r & 0 & 0 \\
0 & 0 & 0 & 0 \\
\cos r & 0 & 0 & 1
\end{array}\right) .
$$

\section{Environment}

We discuss the local channel, in which each subsystem interacts with its own environment and has no communication with others. Then the total evolution of state $\rho_{s}$ can be expressed as [30]

$$
\varepsilon\left(\rho_{s}\right)=\sum_{\mu \nu} M_{\mu}^{1} \otimes \otimes M_{v}^{N} \rho_{S} M_{\mu}^{1+} \otimes \otimes M_{v}^{N+},
$$

where $M_{\mu}^{i}$ are the Kraus operators, $N$ is the number of the subsystems interacted with the environment. In classical computation, the bit flip $0 \leftrightarrow 1$ is the only error that will occur. However, there are the bit flip, the phase flip and the phase-bit flip in quantum computation because of the possibility of the superposition. And the Kraus operators for the three channels are given by [30]

$$
M_{0}=\sqrt{1-p / 2} * 1, M_{1}^{i}=\sqrt{p / 2} \sigma_{i},
$$

where $i=x$ gives us the bit flip, $i=z$ the phase flip, and $i$ $=y$ the phase-bit flip.

\section{Teleportation in an Open System}

Our teleportation model can be described as follow: Alice and Rob share an entangled state at the beginning, then Alice stays stationary, while Rob accelerates uniformly. Meanwhile, the whole system undergoes the same environment, but each subsystem only interacts with its own environment. And a client wants to send a qubit state from Alice to Rob.

From above discussion we know that we can described the evolved state of two particles undergoing local environment as [31]

$$
\varepsilon\left(\rho_{A R}\right)=\sum_{i, j} \Gamma_{i}^{(A)} \Gamma_{j}^{(R)} \rho_{A R} \Gamma_{i}^{(R) \dagger} \Gamma_{j}^{(A) \dagger},
$$

where $\Gamma_{i}^{(k)}(k=A, R)$ are the Kraus operators that describe the noise channels interacted with $A$ and $R$. Now, we will discuss the phase flip, bit flip and bit-phase flip channels, respectively.

\subsection{Phase Flip Channel}

The phase flip channel is a quantum noise process with loss of quantum information but without loss of energy [31-33]. For this channel, the Kraus operators are given by [30-33]

$$
\begin{aligned}
& \Gamma_{0}^{(A)}=\operatorname{diag}\left(\sqrt{1-p_{A} / 2}, \sqrt{1-p_{A} / 2}\right) \otimes 1_{R}, \\
& \Gamma_{1}^{(A)}=\operatorname{diag}\left(\sqrt{p_{A} / 2},-\sqrt{p_{A} / 2}\right) \otimes 1_{R}, \\
& \Gamma_{0}^{(R)}=1_{A} \otimes \operatorname{diag}\left(\sqrt{1-p_{R} / 2},-\sqrt{1-p_{R} / 2}\right), \\
& \Gamma_{1}^{(R)}=1_{A} \otimes \operatorname{diag}\left(\sqrt{p_{R} / 2},-\sqrt{p_{R} / 2}\right),
\end{aligned}
$$

where $p_{A(R)}\left(0 \leq p_{A(R)} \leq 1\right)$ is parameterized time in the channel $A(R)$. For simplicity, we consider the special situation that the decoherence rate is the same in both channels, i.e. $p_{A}=p_{R} \equiv p$.

Because Rob moves with an uniform acceleration, and the whole system undergoes the phase flip channel, using Equations (5) and (8), we obtain the evolved state 


$$
\rho_{A R_{I}}^{e v o}=\frac{1}{2}\left(\begin{array}{cccc}
\cos ^{2} r & 0 & 0 & (p-1)^{2} \cos r \\
0 & \sin ^{2} r & 0 & 0 \\
0 & 0 & 0 & 0 \\
(p-1)^{2} \cos r & 0 & 0 & 1
\end{array}\right)
$$

If we assume that the client wants to send the state $|\psi\rangle=\alpha|0\rangle+\beta|1\rangle$ to Rob, considering the three subsystems as a whole system, then the total initial state comes to $\rho_{\text {in }}=|\psi\rangle\langle\psi| \otimes \rho_{A R_{\mathrm{I}}}^{e v o}$. And the process of the quantum teleportation is: firstly Alice performs CNOT and Hadamard gate operation on the first two qubits, i.e., $\rho^{\prime}=(H \otimes 1 \otimes 1)(C N O T \otimes 1) \rho_{\text {in }}(C N O T \otimes 1)^{\dagger}(H \otimes 1 \otimes 1)^{\dagger} ;$ then she measures the two qubits in Z-basis. The total state, after her measurement, will collapse to $|i j\rangle\langle i j| \otimes \rho_{I}^{i j} \quad$ with the probability of

$$
p_{i j}=\operatorname{Tr}\left(\left\langle i j\left|\rho_{A R_{I}}^{\prime}\right| i j\right\rangle\right)=\frac{1}{4}
$$

where $\rho_{I}^{i j}$ are given by

$$
\begin{aligned}
& \rho_{I}^{i 0}=\frac{1}{4}\left(\begin{array}{cc}
|\alpha|^{2} \cos ^{2} r & (-1)^{i} \alpha \beta^{*}(p-1)^{2} \cos r \\
(-1)^{i} \alpha^{*} \beta(p-1)^{2} \cos r & |\beta|^{2}+|\alpha|^{2} \sin ^{2} r
\end{array}\right), \\
& \rho_{I}^{i 1}=\frac{1}{4}\left(\begin{array}{cc}
|\beta|^{2} \cos ^{2} r & (-1)^{i} \alpha^{*} \beta(p-1)^{2} \cos r \\
(-1)^{i} \alpha \beta^{*}(p-1)^{2} \cos r & |\alpha|^{2}+|\beta|^{2} \sin ^{2} r
\end{array}\right),
\end{aligned}
$$

finally Alice sends the results of the measurement ( $i$ and $j$ ) to Rob by a classical information channel. According to these information, Rob performs the corresponding quantum gate $\left(X^{j} Z^{i}\right)^{-1}=Z^{i} X^{j}$ on the qubit in his possession, and gets $\rho_{I}^{i j}=Z^{i} X^{j} \rho_{I}^{i j}\left(Z^{i} X^{j}\right)^{\dagger}$, i.e.,

$$
\rho_{I}^{i 0}=\rho_{I}^{00}, \rho_{I}^{i 1}=\frac{1}{4}\left(\begin{array}{cc}
|\alpha|^{2}+|\beta|^{2} \sin ^{2} r & \alpha^{*} \beta(p-1)^{2} \cos r \\
\alpha \beta^{*}(p-1)^{2} \cos r & |\beta|^{2} \cos ^{2} r
\end{array}\right)
$$

Then, the fidelities $F_{i j}=\operatorname{Tr}\left(|\psi\rangle\langle\psi| \rho_{I}^{i j}\right)$ can be cal- culated analytically as

$$
\begin{aligned}
& F_{i 0}=2 \alpha^{2} \beta^{2}(p-1)^{2} \cos r+\alpha^{4} \cos ^{2} r+\beta^{4}+\alpha^{2} \beta^{2} \sin ^{2} r, \\
& F_{i 1}=2 \alpha^{2} \beta^{2}(p-1)^{2} \cos r+\beta^{4} \cos ^{2} r+\alpha^{4}+\alpha^{2} \beta^{2} \sin ^{2} r .
\end{aligned}
$$

From Equation (12), it is clear that the fidelities of the teleportation depend on the channel parameter $p$, acceleration parameter $r$ and state parameter $\beta$. In addition, the suitable value of the fidelity should be the minimum of the $F_{i 0}$ and $F_{i 1}$ since in reality Alice will not know the result of the measurement as the quantum state. Hereafter we take the minimum fidelity and plot them in Figures 1 and 2.

Figure 1 shows that the fidelity decreases monotonously as $p$ increases, i.e., the transmission capacity consistently weakened for this channel. Especially, when $\beta^{2}=\frac{1}{2}$ and $p=1$, the fidelity $F=\frac{1}{2}$, which means that the fidelity will converge if the time of the interacttion between the system and environment is long enough, and the information would never completely disappear. However, when $\beta=\frac{1}{3}$ and $\beta=\frac{\sqrt{3}}{2}$, the fidelities don't gather even the environment interacts for infinite time.

Figure 2 shows that the fidelity of the teleportation has a symmetric point $\beta^{2}=1 / 2$. It is interest to note that the fidelity is independent on $p$ when $\beta^{2}=0$ or $\beta^{2}=1$, which means that as the transmitted state is a single bit the environment has no effect on the teleportation.

\subsection{Bit Flip Channel}

For the bit flip channel, the Kraus operators are

$\Gamma_{0}^{(A)}=\operatorname{diag}(\sqrt{1-p / 2}, \sqrt{1-p / 2}) \otimes 1_{R}$

$\Gamma_{1}^{(A)}=\sqrt{p / 2} \sigma_{x}^{(A)} \otimes 1_{R}$

$\Gamma_{0}^{(R)}=1_{A} \otimes \operatorname{diag}(\sqrt{1-p / 2}, \sqrt{1-p / 2})$

and $\Gamma_{1}^{(R)}=1_{A} \otimes \sqrt{1-p / 2} \sigma_{x}^{(R)}$ [30-33]. By using Equations (5) and (8), the initial state evolves to 


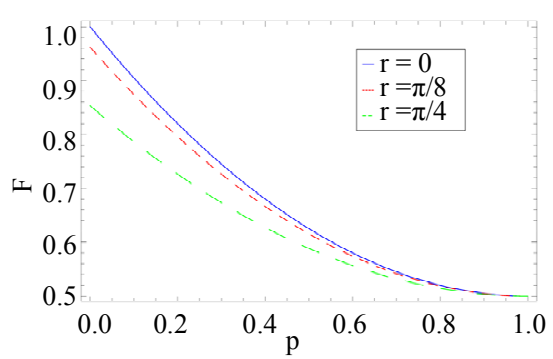

(a)

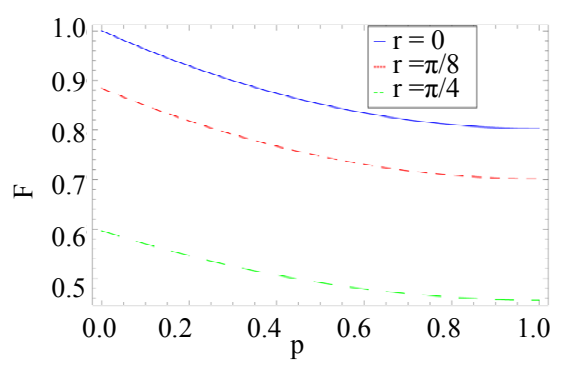

(b)

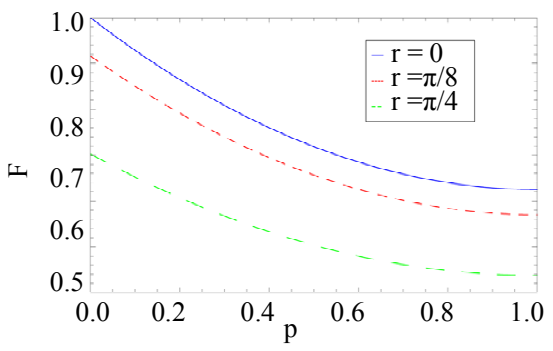

(c)

Figure 1. Fidelity as a function of $p$ with some fixed acceleration parameters $r=0$ (blue curve), $r=\pi / 8$ (red curve) and $r=\pi / 4$ (green curve), and initial state parameters $\beta=\frac{1}{\sqrt{2}}$ (left), $\beta=\frac{1}{3}$ (middle), $\beta=\frac{\sqrt{3}}{2}$ (right) when the system under the phase flip channel.

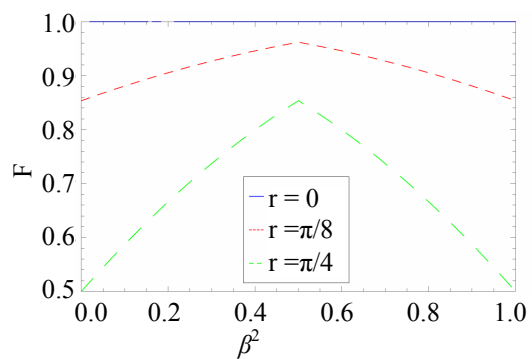

(a)

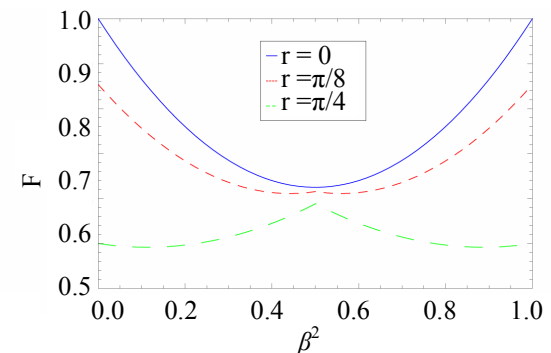

(b)

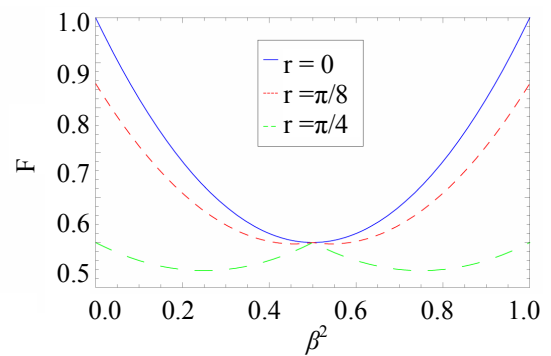

(c)

Figure 2. Fidelity as a function of $\beta^{2}$ with some fixed acceleration parameters $r=0$ (blue curve), $r=\pi / 8$ (red curve) and $r=\pi / 4$ (green curve) when $p=0$ (left), $p=\frac{1}{2}$ (middle) and $p=1$ (right) as the system under the phase flip channel.

$$
\rho_{A R_{I}}^{e v o}=\frac{1}{4}\left(\begin{array}{cccc}
\frac{1}{2} B_{1} & 0 & 0 & (2+p(p-2)) \cos r \\
0 & -\frac{1}{2} B_{2} & -p(p-2) \cos r & 0 \\
0 & -p(p-2) \cos r & -\frac{1}{2} B_{3} & 0 \\
(2+p(p-2)) \cos r & 0 & 0 & \frac{1}{2} B_{4}
\end{array}\right),
$$

with

$$
\begin{aligned}
& B_{1}=2+(p-1) p+(2+(p-3)) p \cos 2 r \\
& B_{2}=(p-2)(1+p+(p-1) \cos 2 r) \\
& B_{3}=p(p-3+(p-1) \cos 2 r)
\end{aligned}
$$$$
\text { and } B_{4}=4+(p-3) p+(p-1) p \cos 2 r \text {. }
$$

Analogous analysis as the subsection A, Rob obtains the state $\rho_{I}^{i j}$

$$
\rho_{I}^{i 0}=\frac{1}{4}\left(\begin{array}{cc}
B_{1} \alpha^{2}-B_{3} \beta^{2} & 4 \alpha \beta \cos r \\
4 \alpha \beta \cos r & B_{4} \beta^{2}-B_{2} \alpha^{2}
\end{array}\right)
$$

or

Then the fidelities $F_{i j}$ are

$$
\begin{aligned}
& F_{i 0}=\frac{1}{4}\left(\left(8-B_{2}-B_{3}\right) \alpha^{2} \beta^{2}+B_{4} \beta^{4}+B_{1} \alpha^{4}\right), \\
& F_{i 1}=\frac{1}{4}\left(\left(8-B_{2}-B_{3}\right) \alpha^{2} \beta^{2}+B_{4} \alpha^{4}+B_{1} \beta^{4}\right) .
\end{aligned}
$$

We plot the fidelity in Figures 3 and 4. From Figure 3, we know that when

$\beta^{2}=\frac{1}{2}$ the fidelity is independent on the environment 


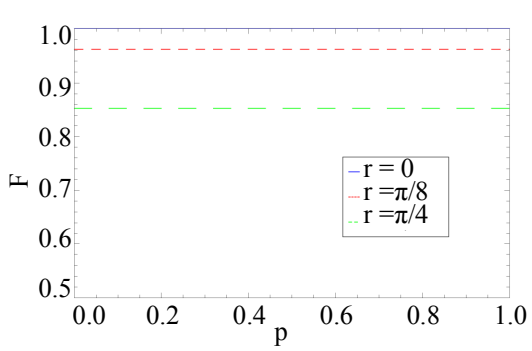

(a)

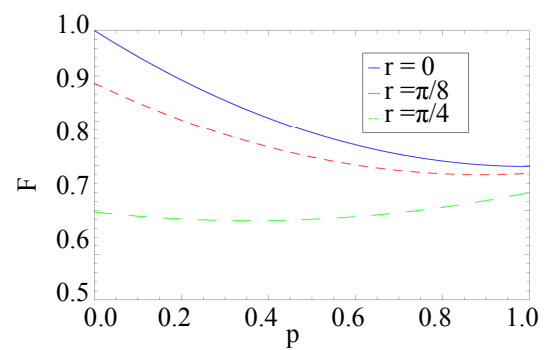

(b)

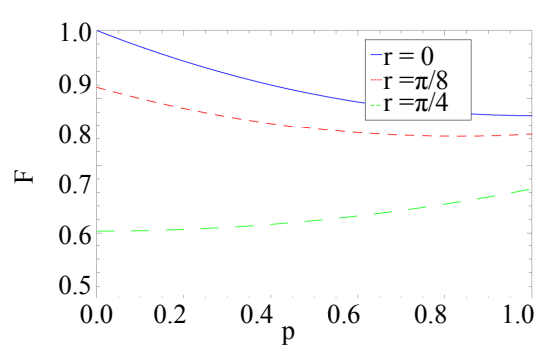

(c)

Figure 3. Fidelity as a function of $p$ with some fixed acceleration parameters $r=0$ (blue curve), $r=\pi / 8$ (red curve) and $r=\pi / 4$ (green curve), and initial state parameters $\beta=\frac{1}{\sqrt{2}}$ (left), $\beta=\frac{1}{3}$ (middle), $\beta=\frac{\sqrt{3}}{2}$ (right) when the system under the bit flip channel.

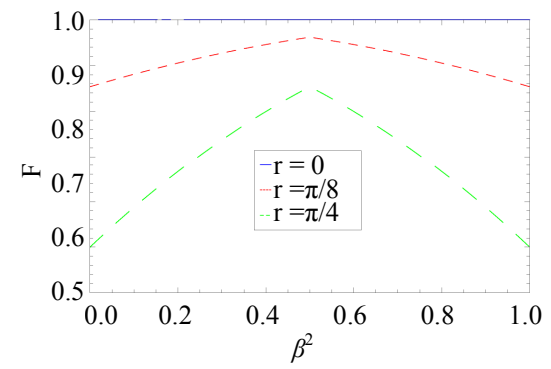

(a)

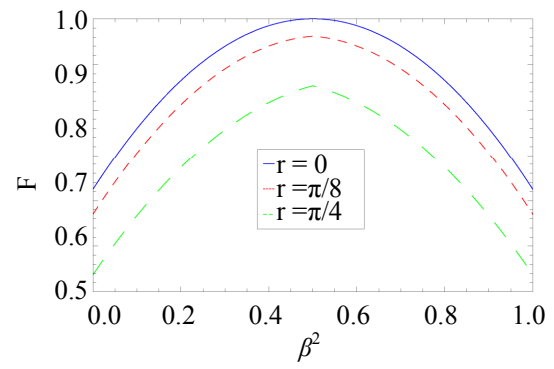

(b)

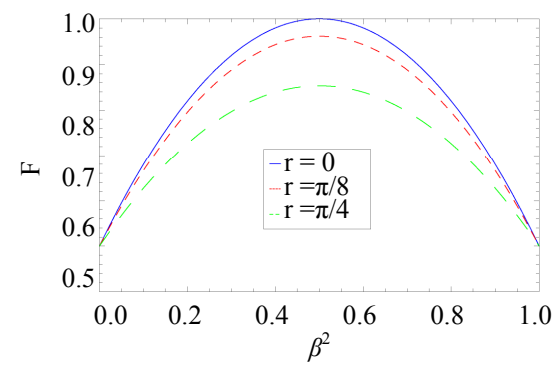

(c)

Figure 4. Fidelity as a function of initial state parameter $\beta^{2}$ with some fixed acceleration parameters $r=0$ (blue curve), $r=\pi / 8 \quad$ (red curve) and $r=\pi / 4 \quad$ (green curve), and the environment parameters $p=0 \quad$ (left), $p=\frac{1}{2} \quad($ middle) and $p=1$ (right) when the system under the bit flip channel.

parameter $p$. However, when $\beta=\frac{1}{3}$ and $\beta=\frac{\sqrt{3}}{2}, \quad$ for $\beta=\frac{1}{3}$ and

the fidelity for $r=0$ decreases monotonously with the increase of $p$, while for $r \neq 0$, it decreases monotonously until

$$
\begin{gathered}
p=\frac{5+23 \cos 2 r}{14(1+\cos 2 r)} \quad \begin{array}{l}
\text { for bit flip channel, the tut } \\
\text { by }
\end{array} \\
p=\left\{\begin{array}{c}
\frac{4 \beta^{2}-1-3 \cos 2 r+4 \beta^{2} \cos 2 r}{2\left(2 \beta^{2}-1\right)(1+\cos 2 r)},\left(\text { for } \beta^{2}<\frac{1}{2}\right), \\
\frac{4 \beta^{2}-3-\cos 2 r+4 \beta^{2} \cos 2 r}{2\left(2 \beta^{2}-1\right)(1+\cos 2 r)},\left(\text { for } \beta^{2}>\frac{1}{2}\right) .
\end{array}\right.
\end{gathered}
$$

for $\beta=\frac{\sqrt{3}}{2}$, then increases monotonously. In general, for bit flip channel, the turning point for $r \neq 0$ is given

The rebound process of the fidelity results from the interaction between Unruh radiation and the quantum decoherence. It is interesting to note that when

$$
\beta^{2} \in\left(\frac{1}{4}, \frac{1}{2}\right) \quad\left(\frac{1}{2}, \frac{3}{4}\right)
$$

the fidelity, provided $r$ is chosen appropriately, will increases consistently by increasing $p$, which means that the environment effect will improve the ability of the transmission for this special case.

In Figure 4, it is obvious that the fidelity is also symmetric around the point $\beta^{2}=\frac{1}{2}$, and the fidelity for $\beta^{2}=\frac{1}{2}$ is always the biggest. Moreover, for single bit 
( $\beta=0$ or $\beta=1)$ the difference of the fidelities of different $r$ cases will becomes smaller and smaller with the increase of $p$. Especially, when $p=1$ they converge to $F=\frac{1}{2}$.

\subsection{Bit-Phase Flip Channel}

For this channel, the Kraus operators are

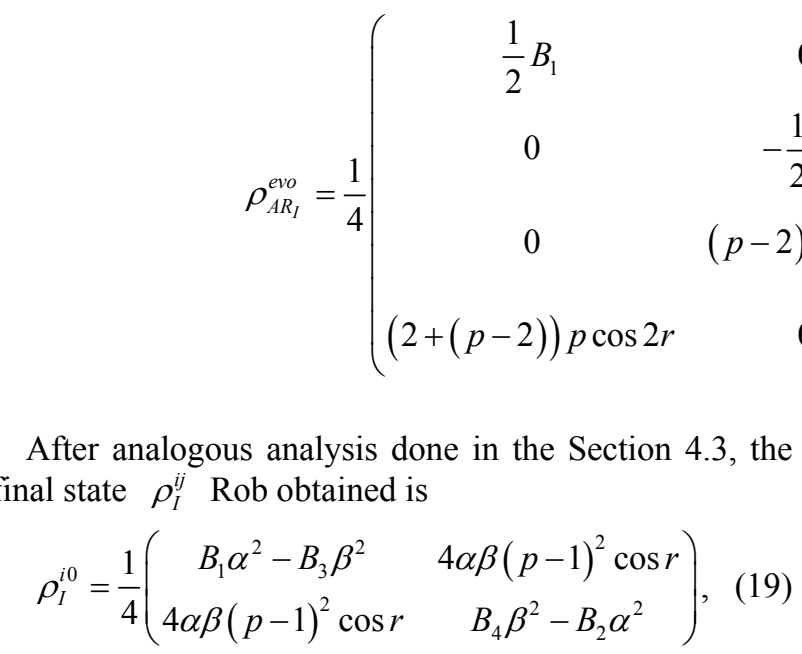

or

$$
\begin{aligned}
& F_{i 0}=\frac{1}{4}\left(\left(8(p-1)^{2}-B_{2}-B_{3}\right) \alpha^{2} \beta^{2}+B_{4} \beta^{4}+B_{1} \alpha^{4}\right), \\
& F_{i 1}=\frac{1}{4}\left(\left(8(p-1)^{2}-B_{2}-B_{3}\right) \alpha^{2} \beta^{2}+B_{4} \alpha^{4}+B_{1} \beta^{4}\right) .
\end{aligned}
$$

$\Gamma_{0}^{(A)}=\operatorname{diag}(\sqrt{1-p / 2}, \sqrt{1-p / 2}) \otimes 1_{R}$

$\Gamma_{1}^{(A)}=\sqrt{p / 2} \sigma_{y}^{(A)} \otimes 1_{R}$

$\Gamma_{0}^{(R)}=1_{A} \otimes \operatorname{diag}(\sqrt{1-p / 2}, \sqrt{1-p / 2})$

and $\Gamma_{1}^{(R)}=1_{A} \otimes \sqrt{p / 2} \sigma_{y}^{(R)}$ [30-33]. Using Equations (5) and (8), the evolved state is given by

$$
\rho_{I}^{i 1}=\frac{1}{4}\left(\begin{array}{cc}
B_{4} \alpha^{2}-B_{2} \beta^{2} & 4 \alpha \beta(p-1)^{2} \cos r \\
4 \alpha \beta(p-1)^{2} \cos r & B_{1} \beta^{2}-B_{3} \alpha^{2}
\end{array}\right) .
$$

The fidelities $F_{i j}$ are

We plot the fidelity of the teleportation in Figures 5

Cor $\beta^{2}=\frac{1}{2}$, the fidelity is monotonously decrease as For $\beta^{2}=\frac{1}{2}$, the fidelity is monotonously decrease as $p$ increases. That is to say, the transmitting capacity becomes weaken when the system interacts with the environment. However, provided $r \neq 0$, the fidelity decreases at the beginning, then increases monotonously, and the turning points are $p=\frac{35+128 \cos r+161 \cos 2 r}{2(49+64 \cos r+49 \cos 2 r)} \quad$ for $\quad \beta=\frac{1}{3} \quad$ a n d $p=\frac{2(3 \cos r+\cos 2 r)}{1+6 \cos r+\cos 2 r}$ for $\beta=\frac{\sqrt{3}}{2}$, respectively. In general, for bit-phase flip the turning point for $r \neq 0$ is

$$
p=\left\{\begin{array}{l}
\frac{\left(-16 \beta^{2}\left(\beta^{2}-1\right) \cos r+\left(2 \beta^{2}-1\right)\left(4 \beta^{2}-1+\left(4 \beta^{2}-3\right) \cos 2 r\right)\right) \sec r}{4\left(-4 \beta^{2}\left(\beta^{2}-1\right)+\left(1-2 \beta^{2}\right)^{2} \cos r\right)},\left(\text { for } \beta^{2}<\frac{1}{2}\right), \\
\frac{\left(-16 \beta^{2}\left(\beta^{2}-1\right) \cos r+\left(2 \beta^{2}-1\right)\left(4 \beta^{2}-3+\left(4 \beta^{2}-1\right) \cos 2 r\right)\right) \sec r}{4\left(-4 \beta^{2}\left(\beta^{2}-1\right)+\left(1-2 \beta^{2}\right)^{2} \cos r\right)},\left(\text { for } \beta^{2}>\frac{1}{2}\right) .
\end{array}\right.
$$

It is worthy to point that the turning point could close to $p=0$ but never reach it with any acceleration.

Figure 6 also shows that the fidelity is symmetric around the axis $\beta^{2}=\frac{1}{2}$. And we are interested to note that the fidelity, when $p=1$, is invariant for any $\beta$ and $r$, i.e., when the time of interaction between subsystem and environment is long enough, both the initial state and Rob's acceleration doesn't affect the fidelity any more, it is always $F=\frac{1}{2}$.

\section{Summary}

The quantum teleportation between two relatively accel 


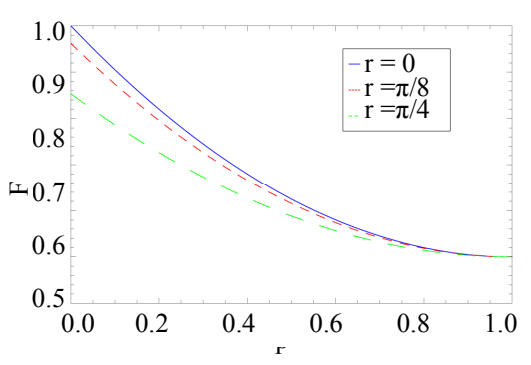

(a)

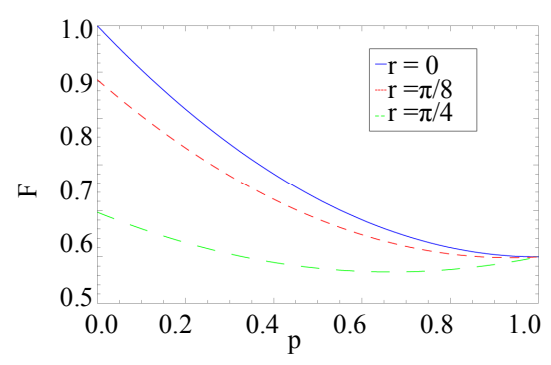

(b)

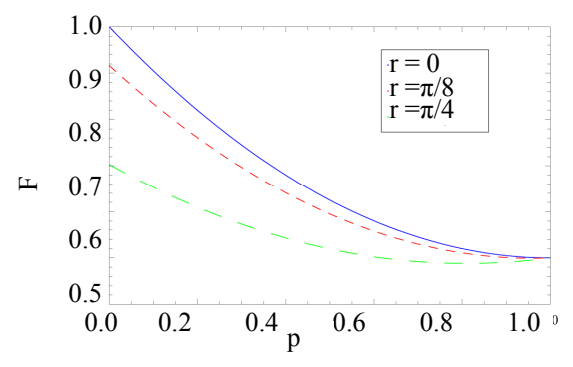

(c)

Figure 5. Fidelity as a function of the environment parameters $p$ with some fixed acceleration parameters $r=0$ (blue curve), $r=\pi / 8$ (red curve) and $r=\pi / 4$ (green curve), and initial state parameters $\beta=\frac{1}{2} \quad$ (left), $\beta=\frac{1}{3}$ (middle), $\beta=\frac{\sqrt{3}}{2}$ (right) when the system under the phase-bit flip channel.

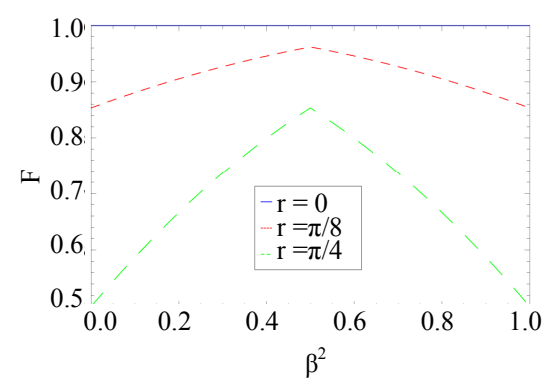

(a)

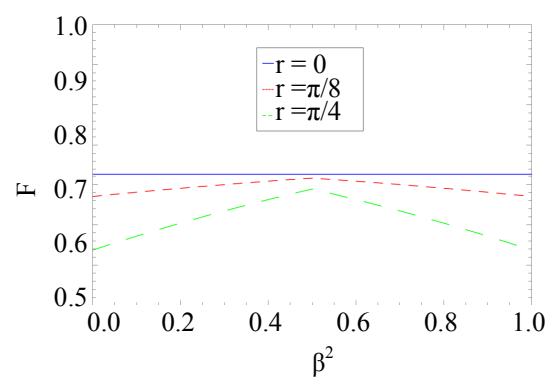

(b)

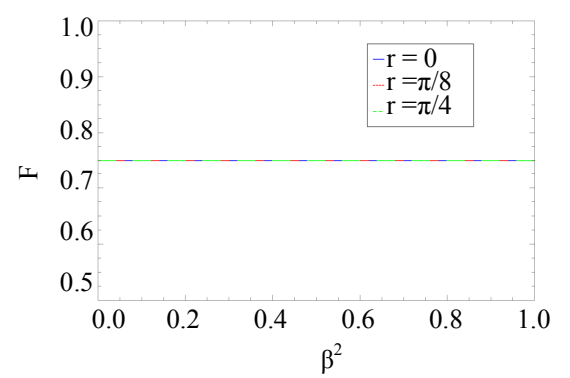

(c)

Figure 6. Fidelity as a function of initial state parameter $\beta^{2}$ with some fixed acceleration parameters $r=0$ (blue curve), $r=\pi / 8$ (red curve) and $r=\pi / 4 \quad$ (green curve), and the environment parameters $p=0 \quad$ (left), $p=\frac{1}{2} \quad$ (middle) and $p=1$ (right) when the system under the phase-bit flip channel.

erated partners undergoing the phase flip, bit flip, and bit-phase flip channels is studied. It was shown that the fidelity of the teleportation depends on the acceleration parameter $r$, environment parameter $p$ and transmitted state parameter $\beta$. By fixing $p$ and $\beta$, the fidelity is consistently decreases with the increase of the acceleration. However, by fixing $r$, the dynamic evolution of the fidelity has different properties, which are listed as follows: 1) For the phase flip channel, the fidelity is monotonously reduced as $p$ increases. When $\beta^{2}=\frac{1}{2}$, the fidelity will converge to $F=\frac{1}{2}$ after a long time of the interaction between the system and the environment; 2) For the bit flip channel, when $\beta^{2}=\frac{1}{2}$, the fidelity of the teleportation is not affected by the environment at all. But when $\beta^{2} \neq \frac{1}{2}$, the fidelity will emerge a turning point at the $p$ axis. Before the point the fidelity decreases monotonously and after that the fidelity increases monotonously. However, the fidelity decreases all the time if Rob is not accelerated. It should be noted out that, provided $r$ is chosen appropriately, the fidelity can increase monotonously with the increase of $p$ when $\beta^{2} \in\left(\frac{1}{4}, \frac{1}{2}\right)\left(\frac{1}{2}, \frac{3}{4}\right)$; And 3) for the phase-bit channel, all the fidelities converge to one point $F=\frac{1}{2}$ for any acceleration and any transmission state parameter $\beta$ if the time of the interaction between the system and the environment is long enough. The fidelity is also monotonously decreasing when $\beta^{2}=\frac{1}{2}$ or the acceleration is zero in this channel.

In addition to that, undergoing any of the three environment the fidelity is always symmetric about $\beta^{2}=\frac{1}{2}$ as it is a function of the transmission state parameter $\beta$. And it, as $\beta^{2}=0$ or $\beta^{2}=1$, is constant for the phase flip channel, while it is constant with $\beta^{2}=\frac{1}{2}$ for the bit 
flip channel, which indicates that there are the different characters between these environments.

\section{Acknowledgements}

This work was supported by the National Natural Science Foundation of China under Grant No. 11175065, 10935013; the National Basic Research of China under Grant No. 2010CB833004; the SRFDP under Grant No. 20114306110003; PCSIRT, No. IRT0964; the Hunan Provincial Natural Science Foundation of China under Grant No 11JJ7001; and Construct Program of the National Key Discipline.

\section{REFERENCES}

[1] A. Einstein, B. Podolsky and N. Rosen, "Can QuantumMechanical Description of Physical Reality Be Considered Complete?" Physical Review, Vol. 47, No. 10, 1935, p. 777. doi:10.1103/PhysRev.47.777

[2] R. Horodecki, P. Horodecki, M. Horodecki and K. Horodecki, "Quantum Entanglement," Reviews of Modern Physics, Vol. 81, No. 2, 2009, p. 865. doi:10.1103/RevModPhys.81.865

[3] C. H. Bennett, G. Brassard, C. Crpeau, R. Jozsa, A. Peres and W. K. Wootters, "Teleporting an Unknown Quantum State via Dual Classical and Einstein-Podolsky-Rosen Channels," Physical Review Letters, Vol. 70, No. 13, 1993, p. 1895. doi:10.1103/PhysRevLett.70.1895

[4] Y. Yeo and W. K. Chua, "Teleportation and Dense Coding with Genuine Multipartite Entanglement," Physical Review Letters, Vol. 96, No. 6, 2006, Article ID: 060502. doi:10.1103/PhysRevLett.96.060502

[5] G. Gordon and G. Rigolin, "Generalized Teleportation Protocol," Physical Review A, Vol. 73, No. 4, 2006, Article ID: 042309. doi:10.1103/PhysRevA.73.042309

[6] L. Mista and R. Filip, "Optimal Partial Deterministic Quantum Teleportation of Qubits," Physical Review A, Vol. 71, No. 4, 2005, Article ID: 022319.

[7] M. Fujii, "Continuous-Variable Quantum Teleportation with a Conventional Laser," Physical Review A, Vol. 68, No. 5, 2003, Article ID: 050302. doi:10.1103/PhysRevA.68.050302

[8] D. Bouwmeester, J. Pan, K. Mattle, M. Eibl, H. Weinfurter and A. Zeilinger, "Experimental Quantum Teleportation," Nature, Vol. 390, No. 6660, 1997, pp. 575-579. doi: $10.1038 / 37539$

[9] P. M. Alsing, "Teleportation in a Non-Inertial Frame," Journal of Optics B: Quantum and Semiclassical Optics, Vol. 6, No. 8, 2004, p. S834. doi:10.1088/1464-4266/6/8/033

[10] P. M. Alsing and G. J. Milburn, "Teleportation with a Uniformly Accelerated Partner," Physical Review Letters, Vol. 91, No. 18, 2003, Article ID: 180404. doi:10.1103/PhysRevLett.91.180404

[11] M. R. Hwang, D. Park and E. Jung, "Tripartite Entanglement in a Noninertial Frame," Physical Review A, Vol. 83,
No. 1, 2001, Article ID: 012111.

[12] B. L. Hu, A. Roura and S. Shresta, "Vacuum Fluctuations and Moving Atoms/Detectors: From the Casimir? Polder to the Unruh? Davies? DeWitt? Fulling Effect," Journal of Optics B: Quantum and Semiclassical Optics, Vol. 6, No. 8, 2004, p. S698. doi:10.1088/1464-4266/6/8/011

[13] Q. Pan and J. Jing, "Degradation of Nonmaximal Entanglement of Scalar and Dirac Fields in Noninertial Frames," Physical Review A, Vol. 77, No. 2, 2008, Article ID: 024302. doi:10.1103/PhysRevA.77.024302

[14] M. Montero and E. Martin-Martinez, "The Entangling Side of the Unruh-Hawking Effect," Journal of High Energy Physics, Vol. 2011, No. 7, 2011, p. 6.

[15] J. Wang, J. Deng and J. Jing, "Classical Correlation and Quantum Discord Sharing of Dirac Fields in Noninertial Frames," Physical Review A, Vol. 81, No. 5, 2010, Article ID: 052120. doi:10.1103/PhysRevA.81.052120

[16] J. Wang and J. Jing, "Multipartite Entanglement of Fermionic Systems in Noninertial Frames," Physical Review $A$, Vol. 83, No. 2, 2011, Article ID: 022314. doi:10.1103/PhysRevA.83.022314

[17] X. H. Ge and Y. G. Shen, "Teleportation in the Background of Schwarzschild Space? Time," Physics Letters B, Vol. 606, No.1-2, 2005, p. 184. doi:10.1016/j.physletb.2004.11.067

[18] X. H. Ge and S. P. Kim, Class. "Quantum Entanglement and Teleportation in Higher Dimensional Black Hole Spacetimes," Classical and Quantum Gravity, Vol. 25, No. 7, 2008, Article ID: 075011. doi:10.1088/0264-9381/25/7/075011

[19] X. H. Ge and Y. G. Shen, "Quantum Teleportation with Sonic Black Holes," Physics Letters B, Vol. 623, No. 1-2, 2005, p. 141. doi:10.1016/i.physletb.2005.07.036

[20] Q. Pan and J. Jing, "Hawking Radiation, Entanglement, and Teleportation in the Background of an Asymptotically Flat Static Black Hole," Physical Review D, Vol. 78, No. 6, 2008, Article ID: 065015. doi:10.1103/PhysRevD.78.065015

[21] B. N. Esfahani, M. Shamirzai and M. Soltani, "Reduction of Entanglement Degradation in Einstein-Gauss-Bonnet gravity," Physical Review D, Vol. 84, No. 2, 2011, Article ID: 025024

[22] D. Giulini, E. Joos, C. Kiefer, J. Kupsch, I. O. Stamatescu and H. D. Zeh, "Decoherence and the Appearence of a Classical World in Quantum Teory," Springer, Berlin, 1996, pp. 44-107.

[23] M. A. Schlosshauer, "Decoherence and the Quantum-toClassical Transition," Springer, Berlin, 2007.

[24] M. Brune, E. Hagley, J. Dreyer, X. Maitre, A. Maali, C. Wunderlich, J. M. Raimond and S. Haroche, "Observing the Progressive Decoherence of the Meter in a Quantum Measurement," Physical Review Letters, Vol. 77, No. 24, 1996, p. 4887. doi:10.1103/PhysRevLett.77.4887

[25] C. J. Myatt, B. E. King, Q. A. Turchette, C. A. Sackett, D. Kiepinski, W. M. Itano, C. Monroc and D. J. Wineland, "Decoherence of Quantum Superpositions through Coupling to Engineered Reservoirs," Nature, Vol. 403, No. 6767, 2000, p. 269. doi:10.1038/35002001 
[26] M. Aspachs, G. Adesso and I. Fuentes, "Optimal Quantum Estimation of the Unruh-Hawking Effect," Physical Review Letters, Vol. 105, No. 15, 2010, Article ID: 151301. doi:10.1103/PhysRevLett.105.151301

[27] E. Martin-Martinez, L. J. Garay and J. Leon, "Unveiling Quantum Entanglement Degradation near a Schwarzschild Black Hole," Physical Review D, Vol. 82, No. 6, 2010, Article ID: 064006.

[28] E. Martin-Martinez, L. J. Garay and J. Leon, "Quantum Entanglement Produced in the Formation of a Black Hole," Physical Review D, Vol. 82, No.6, 2010, Article ID: 064028. doi:10.1103/PhysRevD.82.064028

[29] J. Wang and J. Jing, "System-Environment Dynamics of X-Type States in Noninertial Frames," Annals of Physics, Vol. 327, No. 2, 2012, p. 283 doi:10.1016/j.aop.2011.10.002

[30] A. Salles, F. de Melo, M. P. Almeida, M. Hor-Meyll, S. P. Walborn, P. H. Souto Ribeiro and L. Davidovich, "Ex- perimental Investigation of the Dynamics of Entanglement: Sudden Death, Complementarity, and Continuous Monitoring of the Environment," Physical Review A, Vol. 78, No. 2, 2008, Article ID: 022322. doi:10.1103/PhysRevA.78.022322

[31] J. Maziero, L. C. Celeri, R. M. Serra and V. Vedral, "Classical and Quantum Correlations under Decoherence," Physical Review A, Vol. 80, No. 4, 2009, Article ID: 044102. doi:10.1103/PhysRevA.80.044102

[32] M. A. Nielsen and I. L. Chuang, "Quantum Computation and Quantum Information," Cambridge University Press, Cambridge, 2000, pp. 425-427.

[33] J. Maziero, T. Werlang, F. Fanchini, L. C. Celeri and R. M. Serra, "System-Reservoir Dynamics of Quantum and Classical Correlations," Physical Review A, Vol. 81, No. 2, 2010, Article ID: 022116. doi:10.1103/PhysRevA.81.022116 